\title{
Traduire
}

Revue française de la traduction

$230 \mid 2014$

À la croisée du texte et de l'image

\section{Traduire les nuages}

\section{Giovanni Zucca}

Traducteur : Vanessa De Pizzol

\section{OpenEdition}

Journals

\section{Édition électronique}

URL : http://journals.openedition.org/traduire/631

DOI : $10.4000 /$ traduire.631

ISSN : 2272-9992

Éditeur

Société française des traducteurs

Édition imprimée

Date de publication : 15 juin 2014

Pagination : 87-92

ISSN : 0395-773X

Référence électronique

Giovanni Zucca, «Traduire les nuages », Traduire [En ligne], 230 | 2014, mis en ligne le 15 juin 2016, consulté le 01 mai 2019. URL : http://journals.openedition.org/traduire/631 ; DOI : 10.4000/ traduire.631 


\section{Traduire les nuages(1)}

\section{Giovanni Zucca}

Cet article décrit l'expérience de l'auteur dans la traduction de la BD du français à l'italien ; un rapport affectif que celui avec la " bédé ", devenu professionnel, sans pour autant perdre, bien au contraire, la composante émotive et amoureuse pour les "nuages parlants".

La première fois, ce fut avec le Corrierino, le mythique et inoubliable Corriere dei Piccoli(2).

La première fois avec les $\mathrm{BD}$, je veux dire.

J'avais six ou sept ans, je crois. Je lisais et regardais les images, en même temps, et je me disais qui sait, peut-être qu'un jour je serai moi aussi capable d'en " faire ", des BD - paroles et dessins, dessins et paroles, une petite magie qui se répète, dans l'espace restreint, infini, de la vignette, puis de la planche...

Les années passent, et mon parcours de lecteur compte toujours la BD dans ses centres d'intérêt. Le Corriere dei piccoli devient dei Ragazzi, puis disparaît (il a d'abord publié, parmi mille autres auteurs, Hugo Pratt et Sergio Toppi, et les histoires de Lucky Luke, Michel Vaillant et Bernard Prince) ; suivent Mickey et les Donalds de Disney, et Soldino(3) et Kriminal(4) (quant aux BD érotiques je n'en soufflerai mot, par respect : officiellement en Italie personne n'en achetait, on se les procurait chez le coiffeur...), les éternelles séries qu'on trouvait en kiosque, publiées par les éditions Sergio Bonelli, des incontournables chez nous : Tex, Zagor, Mister

(1) N.d.T : nuvole signifie " nuages " en italien. Ici, référence implicite et poétique à nuvolette (littéralement " petits nuages ") qui dans la terminologie de la BD signifie "bulles".

(2) http://it.wikipedia.org/wiki/Corriere dei Piccoli

(3) N.d.T : Toto 1er (=Soldino), gamin adopté par une grand-mère, Tartine Mariol (en italien nonna Abelarda). BD italienne créée par Giulio Chierchini en 1953, agrémentée du personnage de Toto, créé plus tard par Carpi.

(4) N.d.T : BD noire italienne, créée en 1964 par Max Bunker (de son vrai nom Luciano Secchi, scénario) et Magnus (de son vrai nom Roberto Raviola, dessin). 
No, Dylan Dog. .. Sans oublier Corto Maltese, les Scorpioni del Deserto(5), et Ken Parker(6). Un brin de super-héros, mais raisonnablement - je n'ai jamais eu un bon feeling avec Superman et Spiderman.

En attendant j'écris et je publie des récits, mais les BD non, sapristi, je n'ai jamais eu l'occasion d'en "faire".

Quoique, quoique... Un beau jour je tombe sur un jeune homme, un p'tit gars à la houppette et au pantalon de zouave. II est très connu dans le monde entier, et s'est retrouvé (et il va s'y retrouver une nouvelle fois, avec la complicité de Steven Spielberg et Peter Jackson) sur grand écran.

II s'appelle Tintin. Sauf qu'entre-temps je suis devenu traducteur d'édition. Et je ne dois pas uniquement le lire : je dois (re)traduire en italien tous les albums du jeune reporter-détective.

Cette proposition me coupe le souffle pendant un moment. C'est un défi, une sacrée responsabilité. Je la saisis avec gravité - et en même temps une joyeuse inconscience.

Je vais inscrire des mots dans les nuages!

L'aventure se prolonge dans le temps avec au beau milieu, d'autres histoires, écritures, traductions.

J'ai déjà traduit de très nombreux livres, mais Tintin est ma première approche de la traduction de $\mathrm{BD}$, une approche astreignante et prestigieuse : le texte ne se pose pas librement sur la page blanche, mais il est circonscrit par ces bulles qu'enfant j'espérais remplir un jour de mes propres mots.

Objectif à moitié atteint, les mots sont bien les miens mais en partant de ceux d'Hergé, l'auteur. Je dirais même plus, l'Auteur.

Je lis, je réfléchis, je me lance, mon premier essai (Le secret de la Licorne) est à revoir presque entièrement, je n'en ai pas bien évalué les contours, pour dire les choses.

Parce que je dois me mesurer par exemple aux très célèbres insultes du capitaine Haddock, qui mélangent straccioni (va-nu-pieds) et cretini transalpini (crétins des Alpes) à mammalucchi (bachi-bouzouks) et facce da ananas (moules à gaufres) ; à l'omniprésent et dans de nombreux cas proliférant "Mille sabords!", qui deviennent bombarde, par assonance phonétique, longueur quasi identique - il y a des espaces à respecter, notamment dans les BD comme Tintin, où le texte est très présent - et champ sémantique proche : le "sabord " est le portello di murata des voiliers du xve siècle et bien avant, d'où une fois ouvert, sort le canon, et la

(5) N.d.T : Série de BD créée par Hugo Pratt, qui a pour cadre l'Afrique orientale des années 40.

(6) N.d.T : Personnage dont le nom complet est Kenneth Parker, créé par Giancarlo Berardi (scénario) et Ivo Milazzo (dessin) en 1977, pour une BD du genre Western. 
bombarda est une sorte de canon, même s'il ne s'agit pas, que je sache, d'artillerie navale mais terrestre, destinée au siège, du reste il est clair que rendre

"Mille sabords!"

par

\section{Per mille portelli di murata!}

reste plutôt inenvisageable, à plus d'un titre (même abrégée en : Per mille portelli ! on a une assonance que je trouve très gênante, et cela évoque les sportelli [portières] et même, pire encore, les porcelli [porcelets], Ahrrrr !!!).

En revanche Mille sabords ! = Per mille bombarde ! ça peut marcher, c'est pas mal.

L'aventure - je devrais dire en réalité l'histoire d'amour - avec Tintin, Haddock, le professeur Tournesol et les impayables Dupond et Dupont se poursuit (je dirais même plus... se suit pour !) au gré de quelques réflexions sur le choix des noms et leur italianisation possible. Avec le consultant de l'éditeur pour la traduction, le " bédélogue " Gianfranco Goria, grand expert de tout l'univers d'Hergé, nous décidons que le professeur Tournesol deviendra littéralement Girasole, en tenant compte de la variante possible Girasol, sans " e " final (variante écartée par la suite), tandis que pour les deux policiers farfelus, qui en anglais comme on sait sont devenus Thompson et Thomson, avait surgi la possibilité d'un Derossi et Derosso, qui ont la même initiale, rappellent l'un des noms italiens les plus répandus, c'est-à-dire Rossi, mais ont la même longueur - maintenant que je l'écris, je pense à une autre solution possible qui aurait pu être Derossi et Degrossi...

Inutile réflexion venue après coup, car on a privilégié la fidélité à l'original, et on a fait le choix de garder le double Dupond avec $t$ final.

L'aventure de la traduction italienne de Tintin (qui n'est pas la première, mais la première complète, car dans les précédentes traductions il manquait soit le premier épisode en noir et blanc de 1929, soit le dernier Tintin et l'Alph'art, demeuré inachevé, à l'état d'ébauche, du fait de la disparition de l'auteur) se prolonge avec différents faux départs jusqu'à il y a quelques mois et la sortie du film de Spielberg.

Entre-temps, entre deux livres, traduire la BD ou le graphic novel, qui en France s'appelle je crois roman graphique, est devenu pour moi un rendez-vous professionnel que j'aime et que j'apprécie tout particulièrement : plus bref en général que pour un livre, je n'ai plus eu entre les mains des sagas d'envergure qui embrassent tout comme celle de notre jeune fouineur porte-étendard de la " ligne claire ", mais j'ai traduit des histoires et des albums, parfois de genre (les premières aventures de la série Le Tueur, de Matz et Jacamon, devenu I/ killer, mot anglais qui dans l'usage a remplacé l'italien sicario ou assassino a pagamento), parfois à la croisée des genres au point de devenir un genre en soi (Les aventures d'Adèle Blanc-Sec de l'immense Jacques Tardi). 
Et il me semble qu'au fur et à mesure s'affine la capacité à gérer le texte en rapport avec l'espace (variable d'une BD à l'autre)(7), à savoir, gérer au mieux, dans les pas de l'auteur, le rapport fondamental entre paroles et image (ça semble une banalité, mais ça ne va pas toujours de soi) ; " convertir " les composantes onomatopéiques (le BOUM ! qui devient BUM !) ; réfléchir aux jeux de mots, qui par moments s'enclenchent dans la bulle d'une vignette et se poursuivent sur deux ou trois vignettes... J'ai en mémoire un exemple de Tintin nel paese dell'oro nero(8), du même ordre que celui que nous citons partiellement ci-dessous, dans lequel un "méchant ", piqué par notre héros, fait semblant d'être fou, en enchaînant une série d'affirmations sans queue ni tête, à mi-chemin entre locutions et dictons, où le dernier mot de chaque phrase lance la suivante, sur trois vignettes de suite.

Quel chien... Dent... Hé ! Hé ! Chiendent... Danton et Robespierre... Qui roule n'amasse pas mousse ! Hé ! Hé ! Pas mal, n'est-ce pas ? Et ce n'est pas tout !...

Cane... Grande! Eh, eh! Cangrande... Della scala... Mobile, eh, eh, mobile bar, non male, eh?

Un exemple de la manière dont la "fidélité " en traduction peut devenir un concept élastique, se faisant fort d'adapter des dictons en renonçant dès le départ à l'atout de la phonétique, carte que l'italien n'a pas : Dent - Danton en italien devient Dente - Danton, raison pour laquelle en conservant le " chien ", le " cane ", on pouvait jouer sur "Denton " (dentone = grosse dent) mais il fallait dire adieu à l'association Robespierre et " pierre qui roule " (ce qui a ensuite été le cas, pour des raisons d'espace)...., sans compter que cane-dente/cane-dentone ne mène nulle part, de sorte que la route est toute tracée pour Cangrande della Scala, personnage de l'Histoire italienne ayant vécu bien avant les deux protagonistes de la Révolution française, mais qui m'offre ce scala qui devient mobile(9) - dans ce contexte précis, le fait que l'italien contienne un double sens n'a pas grande importance - pour ensuite recevoir les bouteilles de liqueur, les verres et le seau à glace du " bar ".

Cet exemple met en lumière deux éléments d'ordre plus général qui sont, d'une part, le choix d'un usage plus mesuré des points de suspension, dont l'abus en italien est très lourd, et de l'autre, un texte plus court dans l'ensemble - il ne s'agit que d'une seule bulle dans une vignette -

(7) Parfois ce n'est pas un problème, parfois il faut faire attention, pour ne pas mettre en difficulté la personne qui va s'occuper du lettrage car au final il faudra tenir compte du format du volume, qui pourra nécessiter quelques modifications de dernière minute du texte traduit, lesquelles peuvent émaner directement de la rédaction, par ailleurs très fiable.

(8) Tintin au pays de l'or noir.

(9) N.d.T. scala mobile = littéralement " escaliers mobiles ", autrement dit " escalator ", mais c'est aussi un vocabulaire qui concerne l'économie, l'inflation. 
du fait qu'on a recours dans l'édition italienne à une police légèrement plus grande, qui provient d'un lettrage obligeant à garder une marge blanche plus importante entre le texte et les bords de la vignette(10).

Dans un épisode des Aventures d'Adèle Blanc-Sec je me suis également retrouvé devoir adapter un dialogue qui se poursuit sur plusieurs vignettes (car ici selon moi il s'agit d'une adaptation à proprement parler, plutôt que d'une traduction), et je m'en suis servi pour un article destiné à la rubrique La parola al traduttore(11) hébergée par le foisonnant site de Zanichelli, l'un des éditeurs de dictionnaires italiens les plus importants.

Au-delà de mon expérience, qui pour ce qui est de la BD en langue française comprend désormais une cinquantaine d'albums, il me semble pouvoir dire que, de manière plus générale, la traduction de BD connaît depuis quelques années maintenant un essor conséquent et a gagné un meilleur statut tant du point de vue de la qualité que de la reconnaissance, élément essentiel pour notre catégorie professionnelle trop souvent déconsidérée ; par le passé en effet, il n'était pas rare que le colophon des BD traduites ne fasse même pas mention du traducteur - ce fut le cas, mais ce n'est qu'un exemple parmi tant d'autres, avec la saga du lieutenant Blueberry, le célèbre héros de western de Charlier et Giraud, qui du reste a connu au cours des années au moins trois traductions italiennes différentes, contenant des différences significatives qui aujourd'hui encore font l'objet d'études(12). Certains font coïncider ce virage avec la montée en puissance du graphic novel déjà mentionné (n.b. j'emploie délibérément le terme au masculin, en le traduisant de l'anglais novel entendu comme "roman ", mais nombreux sont ceux qui parlent " de la " graphic novel, par assimilation, du fait de sa plus grande brièveté, à la " nouvelle ", au sens américain d'histoire brève) une version, qu'on pourrait définir, adulte, sérieuse, souvent engagée politiquement et par nature politiquement correcte de la BD - comme si (Andrea Plazzi(13), grand expert et traducteur de BD le faisait remarquer), la $\mathrm{BD}$ en soi n'avait pas une dignité culturelle suffisante pour voguer comme une grande et de plein droit dans la vaste mer de la littérature. Tant et si bien que la présence parmi les douze livres candidats à l'édition 2014 du Premio Strega de unastoria, roman graphique signé de Gipi, a suscité quelques perplexités - comment ça, une « $\mathrm{BD}$ » candidate au plus grand prix littéraire italien, c'est du jamais vu! (et en effet, c'est la première fois, en 68 ans d'histoire du prix littéraire). Évidemment, si unastoria gagnait le Strega, ce serait un sacré coup ce dont personnellement je serais plus qu'heureux, d'autant que Gipi en personne, face aux tentatives de " donner des lettres de noblesse "à son travail, a répondu en ces termes :

(10) II serait intéressant de souligner la précision avec laquelle un grand auteur tel qu'Art Spiegelman donne ses instructions concernant le lettrage: http://www.fumettologica.it/galleria/le-istruzioni-per-il-lettering-di-mausdi-art-spiegelman/

(11) http://dizionaripiu.zanichelli.it/la-parola-al-traduttore/2013/05/20/allanima-della-mafia/

(12) http://scuolatraduzionefumetto.wordpress.com/2009/03/16/le-avventure-del-tenente-blueberry-in-traduzioneitaliana/ (premier d'une série d'articles que l'on peut trouver sur le site indiqué).

(13) http://andreaplazzi.wordpress.com/2009/04/20/il-fantasma-del-fumetto/ 
"Q. Mais alors, le terme exact est graphic novel?

R. Le terme pour décrire le moyen que j'utilise en essayant de provoquer des émotions chez le lecteur existe déjà : "BD ». Je n'éprouve aucune nécessité d'en inventer un autre. Car pour moi une bonne $\mathrm{BD}$ est vraiment une œuvre où dessin et texte travaillent de concert pour se traduire en émotions chez le lecteur. Ce travail sur deux voies parallèles et de même importance, je le nomme, moi, "écriture " de la BD. Mais uniquement parce que c'est commode d'utiliser un mot simple. Donc, faisons comme si nous n'avions pas besoin de donner des lettres de noblesse à la BD tout comme nous n'avons pas besoin de le faire pour la littérature ou le cinéma ॥(14).

C'est clair, non ? Ainsi, tandis que j'ai renoncé à l'idée de mettre mes mots dans ces « nuages parlants " blancs (mais certains les ont aussi définis de manière plus poétique "rêveurs "), je traque pour le moment la moindre occasion de traduire les paroles d'autres auteurs qui font un usage magistral du dessin et du texte, comme le dit Gipi, pour transmettre des émotions au lecteur. C'est une manière de conjuguer la passion pour mon travail avec les émotions simples et directes de ce gamin qui encore aujourd'hui feuillette, assis idéalement à mes côtés, les pages colorées du Corriere dei piccoli, pleines de l'enchantement primordial et magique des histoires.

gizeta99@gmail.com giovanni.zucca01@gmail.com

Texte traduit de l'italien par Vanessa De Pizzol

Giovanni Zucca (Piacenza, 1957) est traducteur d'édition, éditeur et auteur. Il collabore avec de grandes maisons d'édition italiennes, a traduit et traduit toujours, du français et de l'anglais, des romans et de la non-fiction, outre la BD. II fait aussi de la relecture de textes d'auteurs italiens. II résume son travail sous la définition d'" artisan éditorial ".

(14) http://www.ilfattoquotidiano.it/2013/12/24/unastoria-il-fumetto-di-gipi-lautore-e-il-mio-certificato-dipacificazione/8246021 